\title{
Differentiation of Lectin Binding Patterns in Developing Oocytes of the Giant Freshwater Prawn, Macrobrachium rosenbergii
}

\author{
Praneet Damrongphol ${ }^{1, *}$ and Boonserm Poolsanguan ${ }^{2}$ \\ 'Department of Biology, Faculty of Science, Mahidol University, Bangkok 10400, Thailand \\ ${ }^{2}$ Center for Biotechnology of Aquaculture, Institute of Science and Technology for Research \\ and Development, Mahidol University, Nakornpatom 73170, Thailand
}

Accepted September 20, 1999

\begin{abstract}
Summary Five different peroxidase-labeled lectins, Helix pomatia agglutinin (HPA), Concanavalin A agglutinin (Con A), Ricinus communis agglutinin (RCA-I), Wheat germ agglutinin (WGA) and Ulex europaeus agglutinin (UEA-I), were used to investigate alterations of the lectin binding patterns during oocyte development of the giant freshwater prawn, Macrobrachium rosenbergii. HPA, Con A and RCA-I binding sites were detected in the cytoplasm of developing oocytes; whereas HPA, WGA and UEA-I binding sites were localized on the oocyte surfaces. Although, HPA binding to the cytoplasm of developing oocytes could be detected at all stages of development, significant differentiation of the binding occurred at vitellogenic stage. Con A or RCA-I binding to the cytoplasm was first detected at early vitellogenic stage but the patterns of binding were altered at later stage. Moreover, HPA, Con A or RCA-I was reactive to different regions of the cytoplasm and produced different types of the reaction products. Formation of new surface components at vitellogenic stage was also implicated. The oocyte surfaces began to be reactive to HPA at early vitellogenic stage and were intensely reactive to HPA, WGA and UEA-I at late vitellogenic stage. Furthermore, affinity of HPA or RCA-I to the nucleolus was also detected at late vitellogenic stage.
\end{abstract}

Key words Lectin, Oocyte, Ovary, Prawn, Macrobrachium rosenbergii.

Various lectin labeling techniques have widely been used to elucidate molecular alterations of the carbohydrate constituents of the cellular components. Extensive works on the binding of lectins to the cell surfaces have been carried out. Studies on the alterations of lectin binding sites during testicular (Kurohmaru et al. 1995, Wine and Chapin 1997) and embryonic (Depaz et al. 1994, Darmico and Jacobs 1995, Miosge et al. 1997) development have been reported. Nevertheless, little work has been done on differentiation of the carbohydrate moieties during oocyte development (Fang and Welsh 1995).

Oocyte maturation and ovarian development of the giant freshwater prawns have been described (Damrongphol et al. 1991, Chang and Shih 1995). Marked change in structure and enormous accumulation of cytoplasmic materials including lipid and yolk contents occur during the process of oocyte development. The present study employs different peroxidase-labeled lectins to investigate differentiation of the lectin binding patterns during oocyte development of the giant freshwater prawn, Macrobrachium rosenbergii.

\section{Materials and methods}

Mature giant freshwater prawns, Macrobrachium rosenbergii, were purchased from the prawn farms in supanburi, Thailand, and they were acclimated in large fiberglass tanks at $26^{\circ} \mathrm{C}$ for 2 days

* Corresponding author, e-mail: scpdr@mahidol.ac.th 
before transferring to separate chambers and laboratory-maintained as previously described (Damrongphol et al. 1991).

Five peroxidase-labeled lectins, Helix pomatia agglutinin (HPA), Concanavalin A agglutinin (Con A), Ricinus communis agglutinin (RCA-I), Wheat germ agglutinin (WGA) and Ulex europaeus agglutinin (UEA-I), and 5 specific inhibitory sugars, $\mathrm{N}$-acetyl- $\alpha$-D-galactosamine for HPA, $\alpha$-D-mannose for Con A, $\beta$-D-galactose for RCA-I, N-acetyl- $\beta$-D-glucosamine for WGA and $\alpha$-Lfucose for UEA-I, were purchased from Sigma Chemical Co. Lectins and inhibitory sugars were dissolved in $0.05 \mathrm{M}$ Tris $\mathrm{HCl}, \mathrm{pH} 7.6$, to appropriate concentrations.

Ovaries at immature stages, which exhibited faint yellowish color and comprised oocytes of all developmental stages (Damrongphol et al. 1991), were dissected from the mature females. The ovaries were cut into pieces and fixed for $24 \mathrm{~h}$ in Bouin's fixative. The specimens were dehydrated through a graded series of ethanol and embedded in paraffin. Blocks were sectioned to $7 \mu \mathrm{m}$ thickness. Deparaffinized sections were incubated with $5 \mu \mathrm{g} / \mathrm{ml} \mathrm{HPA}, 5 \mu \mathrm{g} / \mathrm{ml}$ Con A, $2 \mu \mathrm{g} / \mathrm{ml} \mathrm{RCA}-\mathrm{I}$, $5 \mu \mathrm{g} / \mathrm{ml} \mathrm{WGA}$ or $10 \mu \mathrm{g} / \mathrm{ml}$ UEA-I, for $1 \mathrm{~h}$ at room temperature. Sections were then washed with $0.05 \mathrm{M}$ Tris $\mathrm{HCl}, \mathrm{pH} 7.6$, and incubated with $0.5 \mathrm{mg} / \mathrm{ml} \mathrm{3}$ 3',3' diaminobenzidine (DAB) containing $0.01 \% \mathrm{H}_{2} \mathrm{O}_{2}$ for $15 \mathrm{~min}$. After incubation, the sections were rinsed with deionized water, dehydrated and mounted with permount. Control sections for specificity of the lectins were incubated with various peroxidase-labeled lectins in the presence of $0.3 \mathrm{M}$ of appropriate inhibitory sugars. Sections incubated with $0.5 \mathrm{mg} / \mathrm{ml} \mathrm{DAB}$ containing $0.01 \% \mathrm{H}_{2} \mathrm{O}_{2}$ for $15 \mathrm{~min}$ were prepared as controls for the endogenous peroxidase.

\section{Results}

The faint-yellowish immature ovaries of the giant freshwater prawns contained oogonia and oocytes of all developmental stages. The oogonia were small, relatively round cells and were usually located at the central regions of the ovaries. The previtellogenic oocytes were slightly enlarged and the chromosomes had entered the first meiotic division. While the vitellogenic oocytes were characterized by the diplotene state of the chromosomes. Explicit increase in the mass of the cytoplasm was discrenable in early vitellogenic oocytes. However, further increase in the mass of the cytoplasm accompanying the accumulation of the yolk and the lipid granules were found in late vitellogenic oocytes (Figs. 1, 2).

Variations in the intensity of various lectin binding to different regions of the oogonia and the developing oocytes were evidenced (Table 1). HPA, Con A or RCA-I was reactive to different regions of the cytoplasm and produced different types and patterns of the reaction products at early vitellogenic stage. While HPA, WGA or UEA-I was intensely reactive to the oocyte surfaces at late vitellogenic stage.

The reaction products with HPA in the cytoplasm were granulated and were distributed evenly in the cytoplasm of the oogonia, previtellogenic and early vitellogenic oocytes (Fig. 1). At early vitellogenic stage, the cortical region of the cytoplasm was devoid of the reaction products (Fig. 1). But at late vitellogenic stage, the reaction products became scattered throughout the cytoplasm except for the yolky poles of the oocytes (Fig. 2). HPA binding to the oocyte surfaces was first observed at early vitellogenic stage and became more intense at later stage (Figs. 1, 2). On the other hand, binding to the nuclear envelope was not detected at any stage of the oocyte development, while slight binding to the nucleolus at late vitellogenic stage (Fig. 2) was noticed.

Binding of Con A was found only in the cytoplasm of vitellogenic oocytes (Figs. 3, 4). At early vitellogenic stage, aggregates of the reaction products were found in the cytoplasm closed to the cortical region. The aggregates formed a circular band surrounding the nucleus and became scattered at late vitellogenic stage (Fig. 4).

Binding of RCA-I began to be detected in the cytoplasm of early vitellogenic oocytes (Fig. 5). 

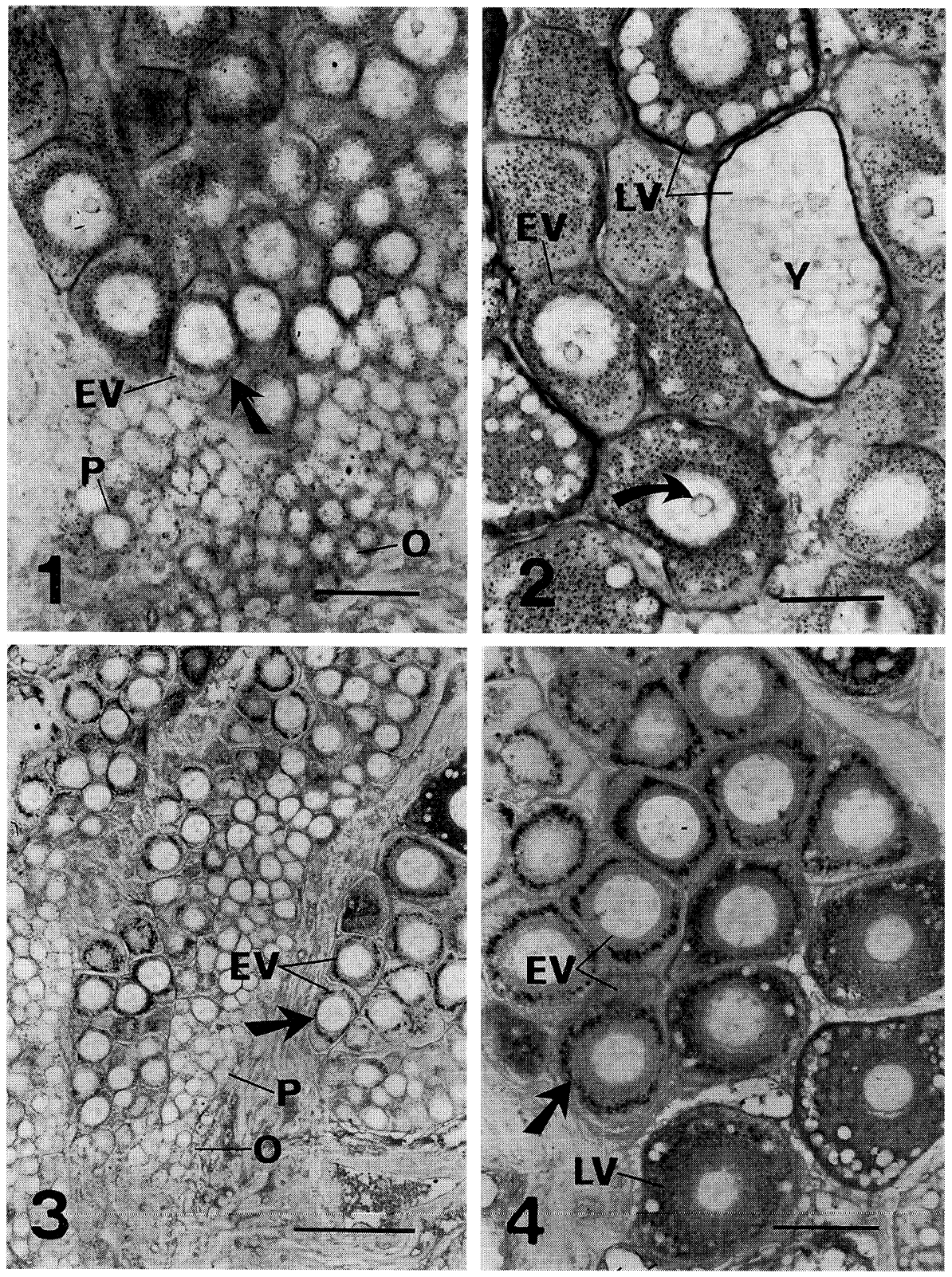

Figs. 1-4. 1) Binding of HPA to the cytoplasm of the oogonia $(\mathrm{O})$, previtellogenic $(\mathrm{P})$ and early vitellogenic (EV) oocytes. The granulated reaction products were evenly distributed throughout the cytoplasm. But at early vitellogenic stage, the products were not detected at the cortical region, and the oocyte surface (arrow) began to react with HPA. Scale bar $=50 \mu \mathrm{m} .2$ ) The granulated reaction products with HPA in the cytoplasm of early (EV) and late vitellogenic (LV) oocytes. At late vitellogenic stage, the yolky pole (Y) was devoid of the reaction products, while the surface was heavily reacted and the nucleolus (arrow) was slightly reacted with HPA. Scale bar $=50 \mu \mathrm{m}$. 3) Binding of Con A (arrow) to the cytoplasm of early vitellogenic (EV) oocytes. Oogonia (O) and previtellogenic (P) oocytes did not show any binding of Con A. Scale bar $=100 \mu \mathrm{m}$. 4) The reaction products with Con A in the cytoplasm of early (EV) and late vitellogenic (LV) oocytes. At early vitellogenic stage, the reaction products (arrow) were aggregated in the cytoplasm closed to the cortical region to form a ring-like structure surrounding the nucleus. But the aggregates were dispersed at late vitellogenic stage. Scale bar $=50 \mu \mathrm{m}$. 
Table 1. Binding of various peroxidase-labeled lectins to the oogonia and the developing oocytes of Macrobrachium rosenbergii

\begin{tabular}{|c|c|c|c|c|c|}
\hline \multirow{2}{*}{$\begin{array}{l}\text { Concentration and type of } \\
\text { peroxidase-labeled lectins }\end{array}$} & \multirow{2}{*}{ Stage of oocyte } & \multicolumn{4}{|c|}{ Extent of binding at various sites } \\
\hline & & Oocyte surface & Cytoplasm & Nuclear envelope & Nucleolus \\
\hline \multirow[t]{4}{*}{$5 \mu \mathrm{g} / \mathrm{ml} \mathrm{HPA}$} & Oogonia & - & ++ & - & - \\
\hline & Previtellogenic & - & ++ & - & - \\
\hline & early vitellogenic & ++ & +++ & - & - \\
\hline & Late vitellogenic & +++ & +++ & - & + \\
\hline \multirow[t]{4}{*}{$5 \mu \mathrm{g} / \mathrm{ml}$ Con A } & Oogonia & - & - & - & - \\
\hline & Previtellogenic & - & - & - & - \\
\hline & early vitellogenic & - & +++ & - & - \\
\hline & Late vitellogenic & - & ++ & - & - \\
\hline \multirow[t]{4}{*}{$2 \mu \mathrm{g} / \mathrm{ml}$ RCA-I } & Oogonia & - & - & - & - \\
\hline & Previtellogenic & - & - & - & - \\
\hline & early vitellogenic & - & +++ & - & - \\
\hline & Late vitellogenic & - & + & - & +++ \\
\hline \multirow[t]{4}{*}{$5 \mu \mathrm{g} / \mathrm{ml} \mathrm{WGA}$} & Oogonia & - & - & - & - \\
\hline & Previtellogenic & - & - & - & - \\
\hline & early vitellogenic & - & - & - & - \\
\hline & Late vitellogenic & +++ & - & - & - \\
\hline \multirow[t]{4}{*}{$10 \mu \mathrm{g} / \mathrm{ml}$ UEA-I } & Oogonia & - & - & - & - \\
\hline & Previtellogenic & - & - & - & - \\
\hline & early vitellogenic & - & - & - & - \\
\hline & Late vitellogenic & +++ & - & - & - \\
\hline
\end{tabular}

+++ intense binding. ++ moderate binding. + slight binding. - no binding.

The reaction products were large precipitates which became dispersed into fine granules at late vitellogenic stage (Fig. 6). No observable RCA-I binding to the oocyte surface or the nuclear envelope was found but strong binding to the nucleolus at vitellogenic stage was observed (Fig. 6).

Beside the intense binding of WGA or UEA-I to the surfaces of late vitellogenic oocytes, no detectable reaction product with WGA or UEA-I at any stage of oocyte development was observed (Figs. 7, 8). The control sections, which incubated with various lectins in the presence of excess amount of specific inhibitory sugars, did not show any reaction with lectins and, thus, exhibited faint images. While the endogenous peroxidase controls produced the brownish backgrounds which were different from the granulated or the precipitated reaction products with lectins.

\section{Discussion}

Considerable alterations of the lectin binding sites in the cytoplasm and on the oocyte surfaces occur during the course of oocyte development in the giant freshwater prawn, Macrobrachium rosenbergii. The presence of terminal $\mathrm{N}$-acetyl- $\alpha$-D-galactosamine, $\alpha$-D-mannose and $\beta$-D-galactose residues in the cytoplasm is revealed by the affinities of HPA, Con A or RCA-I to the cytoplasm of developing oocytes. Likewise, the presence of terminal $\mathrm{N}$-acetyl- $\alpha$-D-galactosamine, $\mathrm{N}$ acetyl- $\beta$-D-glucosamine and $\alpha$-L-fucose on the oocyte surfaces is indicated by the affinities of HPA, WGA or UEA-I to the oocytes surfaces.

Detection of HPA binding in the cytoplasm of oogonia and oocytes at all stages of development in the present study is similar to that reported in the study of developing lancelet oocytes (Fang and Welsh 1995). Thus, N-acetyl- $\alpha$-D-galactosamine residues seem to represent the carbohydrate moieties common for germ cells and may be served as marker for detection of the earlystaged germ cells. 

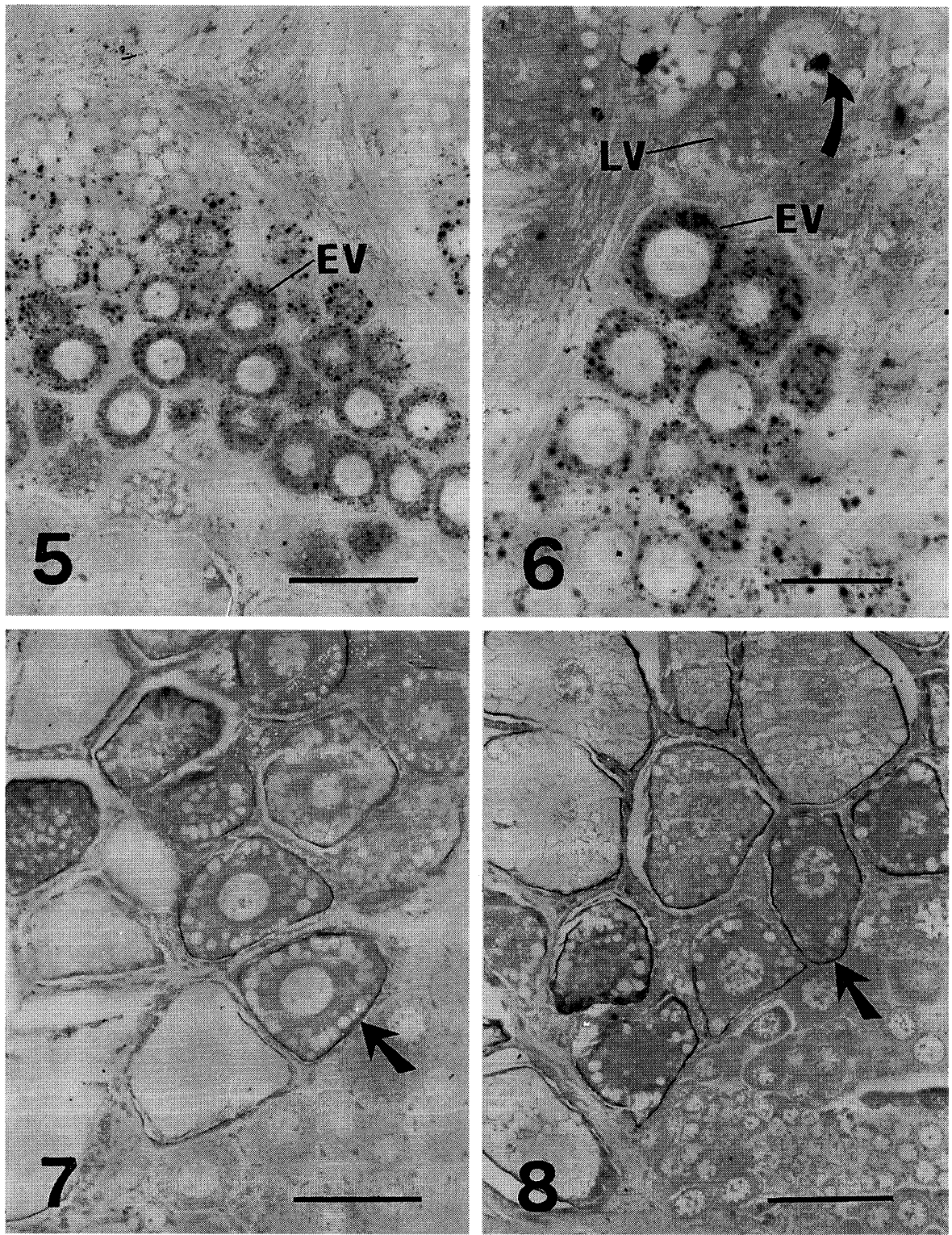

Figs. 5-8. 5) Binding of RCA-I to the cytoplasm of early vitellogenic (EV) oocytes. Scale bar= $100 \mu \mathrm{m}$. 6) Large precipitates of the reaction products with RCA-I in the cytoplasm of early vitellogenic (EV) oocytes. At late vitellogenic (LV) stage, the reaction products became dispersed into fine granules and the nucleolus (arrow) was intensely reactive to RCA-I. Scale bar $=50 \mu \mathrm{m}$. 7) Binding of WGA to the oocyte surface (arrow) of late vitellogenic oocytes. Scale bar $=100 \mu \mathrm{m}$. 8) Binding of UEA-I to the oocyte surface (arrow) of late vitellogenic oocytes. Scale bar $=100 \mu \mathrm{m}$.

HPA, Con A or RCA-I show different patterns of binding and are reactive to different regions of the cytoplasm. HPA binding forms granulated reaction products and is quite uniformly distributed, while Con A binding appears as aggregates forming a circular band surrounding the nucleus and is localized in the cytoplasm closed to the cortical region, while RCA-I binding forms large precipitates and is also relatively uniformly distributed. Con $\mathrm{A}$ is also specific for $\alpha$-D-glucose residues, detection of Con A binding in the cytoplasm of early vitellogenic oocytes may indicate the formation of the glycogen (Murata et al. 1984) at this stage. 
Formation of chitinous envelope is implicated by the presence of $\mathrm{N}$-acetyl- $\beta$-D-glucosamine residues on late vitellogenic oocyte surfaces. This may be the primary protective envelope of the oocyte for further formation of the embryonic envelope which consists of several layers and comprises chitin (Glas et al. 1997). The $\alpha$-L-fucose residues are also the components of late vitellogenic oocyte surfaces. Such terminal residues may involve in the process of gamete interaction, since $\alpha$-L-fucose residues have been found on the external coats of the mammalian oocytes and are suggested to play a role in gamete interaction (Maymon et al. 1994, Lucas et al. 1994).

The present study also indicates that vitellogenic stage is an active state of molecular alterations. Although HPA binding in the cytoplasm is detected since early stage of oocyte development, cytoplasmic alteration by the commencement of Con A and RCA-I binding, the occurrence of new surface components reactive to HPA, WGA and to UEA-I and finally the appearance of RCA-I reactivity at the nucleolus, all occur at vitellogenic stage. The interesting observation of RCA-I affinity to the nucleolus at late vitellogenic stage in the present study correlates with the observation in the lancelet oocytes (Fang and Welsh 1995). The terminal $\beta$-D-galactose residues of the carbohydrate moieties in the nucleolus apparently involve in the alteration of the nucleolar gene activities at late vitellogenic stage.

\section{Acknowledgements}

We would like to thank R. Panya and K. Vejasanit for assistance in preparation of the manuscript. This work was supported by Grant BRG 388001 from the Thailand Research Funds.

\section{References}

Chang, C. F. and Shih, T. W. 1995. Reproductive cycle of ovarian development and vitellogenic profiles in the freshwater prawns, Macrobrachium rosenbergii. Inv. Reprod. Dev. 27: 11-20.

Damico, P. and Jacobs, J. R. 1995. Lectin histochemistry of the Drosophila embryo. Tiss. Cell 27: 23-30.

Damrongphol, P., Eangchuan, N. and Poolsanguan, B. 1991. Spawning cycle and oocyte maturation in laboratory-maintained giant freshwater prawns (Macrobrachium rosenbergii). Aquaculture 95: 347-357.

Depaz, P., Sanchez, A. J., Fernandez, J. G., Garcia, C., Chamorro, C. A. and Anel, L. 1994. Ultrastructural localization of lectin receptors in the preimplantation ovine embryo. Anat. Rec. 240: 537-544.

Fang, Y. Q. and Welsh, U. 1995. A histochemical study of the distribution of lectin binding sites in the developing oocytes of the lancelet Branchiostoma belcheri. Cell Tiss. Res. 280: 427-434.

Glas, P. S., Courtney, L. A., Rayburn, J. R. and Fisher, W. S. 1997. Embryonic coat of the grass shrimp Palaemonetes pugio. Biol. Bull. 192: 231-242.

Kurohmaru, M., Kobayashi, H., Kanai, Y., Hattori, S., Nishida, T. and Hayashi, Y. 1995. Distribution of lectin binding in the testes of the musk shrew, Suncus murinus. J. Anat. 187: 323-329.

Lucus, H., Bercegeay, S., Lependu, J., Jean, M., Mirallie, S. and Barriere, P. 1994. A fucose-containing epitope potentially involved in gamete ineraction on the human zona pellucida. Human. Reprod. 9: 1532-1538.

Maymon, B. B., Maymon, R., Bennun, I., Ghetler, Y., Shalgi, R. and Skutelsky, E. 1994. Distribution of carbohydrate in the zona pellucida of human oocytes. J. Reprod. Fert. 102: 81-86.

Miosge, N., Dresp, W. and Herken, R. 1997. Ultrastructural localization of binding sites for the lectins RCA I, WGA, and LTA in the preimplantation mouse embryo. J. Histochem. Cytochem. 45: 447-453.

Murata, H., Takahashi, N. and Yamada, K. 1984. Effects of digestion with N-oligosaccharide glycopeptidase upon certain lectin-peroxidase-diaminbenzidine reactions of glycoproteins in mammalian and avian tissue. Histochemistry $\mathbf{8 1}$ : 401-407.

Wine, R. N. and Chapin, R. E. 1997. Evaluation of the binding patterns of eleven FITC-conjugated lectins in Fisher 344 rat testes. J. Androl 18: 71-79. 